Research Article

\title{
Elimination of Secondary Neutrons from Laser Proton-Boron Fusion
}

\author{
Heinrich Hora, ${ }^{1,2}$ Shalom Eliezer, ${ }^{3,4}$ and Noaz Nissim (iD ${ }^{4}$ \\ ${ }^{1}$ University of New South Wales, Sydney 2052, Australia \\ ${ }^{2}$ HB11Energy, Freshwater, Sydney 2096, Australia \\ ${ }^{3}$ Polytechnical University, Madrid, Spain \\ ${ }^{4}$ SOREQ Research Centre, Yavne, Israel \\ Correspondence should be addressed to Noaz Nissim; noaznissim@gmail.com
}

Received 26 July 2020; Accepted 13 October 2020; Published 8 April 2021

Academic Editor: Dieter Hoffman

Copyright (c) 2021 Heinrich Hora et al. This is an open access article distributed under the Creative Commons Attribution License, which permits unrestricted use, distribution, and reproduction in any medium, provided the original work is properly cited.

\begin{abstract}
For low carbon energy generation, a very large exchange of electricity generators is existentially vital within the next number of years by power stations preferably at considerably low cost than the present installations. When considering the million times higher nuclear energy per reaction than chemical, the usual hydrogen fusion with abundant boron fuel is used for environmentally clean electricity generators. Instead of usually needed ignition temperatures of hundreds of million degrees Celsius, it is possible to use nonthermal ignition pressures from now available CPA laser pulses. In this non-LTE scheme, there is no need for high compression, the medium of hydrogen-boron-11 temperatures is low, and therefore the bremsstrahlung losses practically do not exist. The neutron, created by secondary reactions, elimination device includes tin and is arranged such that the neutrons are brought to nuclear reactions with the tin. We suggest adding the tin that has proven to be particularly advantageous because of its high effective cross section, and the neutron reactions with tin transform the tin nuclei into stable nuclei with a higher atomic weight.
\end{abstract}

\section{Introduction}

Mechanical power generation is possible by using the steam engine, by electricity, and by using combustion engines with burning coal as the basis for our unique wealth and civilization. The emission of carbon dioxide into the air until 1960 could be tolerated without changing the climate. Since then the carbon emission is five times higher, and during the next 30 years, it is expected to double again. Changing the climate is evident with rising of the average temperature by the green house effect, with measured accelerated rising of the ocean levels from melting of the glaciers, and irreversibly changing of the nature. The European President Dr. von der Leyen at her inauguration to the council on 1 December 2019 mentioned her two aims [1], of which one is that one has not only to prevent the inherent climatic catastrophe but also more severely to realize that this is existentially vital.

One must realize what an extremely monstrous task the solution generation is. In this task, all the renewable energies, photovoltaic, wind energy, and so many others, are of little help. We must realize that, for the next 20 years, to arrive at low carbon energy generation-not to zero-burning carbon will be indispensable, but down to the level of 1960. By the present generation of electricity of about 20 terawatt, it is needed to change to other energy sources. This means we need more than 10,000 new electric power stations, each of the usual costs of nearly 1 billion dollars. This large number of power stations must be drastically less expensive as well as the fuel of the future [2].

For energy, we have a solution at hand: it only needs a modified exploration. We must forget several earlier presumptions, ideologies, and prejudices. The answer is nuclear energy. Since the tremendous discoveries of Lord Rutherford 120 years ago, we know that the energy at a nuclear reaction is in the orders of multiples of 10 million times higher than the energy gained from a chemical reaction such as carbon burning. To get the nuclei to react, one must smash them with accelerators of at least 10 million volts together. When 
such accelerators worked [3], it was then a surprise that reactions happened with light nuclei with energies around $100,000 \mathrm{~V}$. One of these reactions is hydrogen $\mathrm{H}$ with the boron isotope 11 [4], namely, HB11 fusion

$$
\mathrm{H}+{ }^{11} \mathrm{~B}=3^{4} \mathrm{He}+8.7 \mathrm{MeV},
$$

resulting in three helium ${ }^{4} \mathrm{He}$, with an energy spectrum spread between $1.5 \mathrm{MeV}$ and $6 \mathrm{MeV}$ with a maximum in our Sun, four hydrogen nuclei are fused to one helium nucleus (a net reaction) at temperatures above 15 Million of at about $5 \mathrm{MeV} \pm 1 \mathrm{MeV}[5,6]$. When a heavy hydrogen nuclei $\mathrm{D}$ (deuterium) and a superheavy hydrogen $\mathrm{T}$ (tritium) interact, it results in a neutron (n) and an alpha (DT fusion):

$$
\mathrm{D}+\mathrm{T}={ }^{4} \mathrm{He}+\mathrm{n}+17.6 \mathrm{MeV} \text {. }
$$

The nuclear fusion reactions are involved in the energy sources of the myriads of stars, e.g., in our Sun, four hydrogen nuclei are fused to one helium nucleus (a net reaction) at temperatures above 15 Million of ${ }^{\circ} \mathrm{C}$. In the past 60 years, such fusion reactions are tried for power stations on earth, but a solution seems to be many years ahead.

Otto Hahn 1938 by moving the electrically noncharged neutron to very heavy uranium nuclei, causing splitting (fission) and an energy release in the range of $200 \mathrm{MeV}$, discovered another solution. Today, more than $10 \%$ of all global electricity is produced from these nuclear fission power stations. There is the risk-if the control is not perfect-that a meltdown of the reactor as in the USA at Three Mile Island can happen or in Ukraine at Chernobyl with extreme damage. In addition, the handling of the ash is difficult because of the nuclear radiation. What is needed are environmentally clean, safe, electricity generators for abundant nuclear energy and fuel within a short period.

What was interesting from the beginning with the numerous exothermic fusion reactions of light nuclei was that the HB11 reaction (1) resulted primarily only in harmless stable helium and not into radioactive nuclei. We shall consider secondary side reaction by producing a small number of neutrons.

The previous proposals of laser compression of HB11 fusion needs densities up to 100,000 times of the solid state and temperatures of the order of few hundred kiloelectron volts. This situation for laser-driven fusion was changed after the discovery of CPA (chirped pulse amplified) lasers [7] that produce extremely short laser pulses of short duration, less than one picosecond (ps) with extremely ultrahigh powers above petawatt (PW).

With these laser pulses, the first thousand HB11 fusion reactions per laser interaction were measured [8] and then increased above one million [9] and above one billion [10]. The last and simple laser experiment [11] has a gain about one order of magnitude less than the best DT fusion from NIF. This led to the design of a laser fusion reactor (see Figure 16 of [12]) needing a cylindrical trapping of the fusion reactions by kilotesla magnetic fields. The measured high fusion gains profited from the avalanche multiplication by the three generated alpha particles [13].
As recently shown in [14], by using the old cross section for $\mathrm{pB} 11$ [15] at the high temperatures appropriate for the proton-boron-11 fusion, the medium losses more energy by inverse bremsstrahlung that it gains by the nuclear fusion. The novel rates of pB11 fusion (see Figure 4 of [14]) are on the limit of breakeven, but not viable for a fusion reactor. Therefore, our proposal for non-LTE fusion, without heating to high temperatures, is mandatory for a $\mathrm{pB} 11$ fusion reactor [16-18]. These reactors are environmentally clean with respect to nuclear radiation and safe. The initiation of the ignition of the HB11 fusion reaction is possible without needing temperatures of few hundred million degrees Celsius. The CPA laser pulses of picosecond duration and powers above petawatt produce nonthermal pressures above $6 \times 10^{12} \mathrm{~J} / \mathrm{cm}^{3}$ for initiating the ignition of the HB11 nuclear fusion.

\section{Elimination of Secondary Neutrons}

To the question of generation of neutrons by secondary reactions in the spherical $\mathrm{HB} 11$ reactor [12], their sufficient elimination by neutron capture with stable daughter nuclei is used [19]. While HB11 fusion is neutron-free, a secondary reaction occurs by the alpha particles reacting with the boron-11 nuclei present in the fuel, creating harmless stable nitrogen and a neutron. This reaction,

$$
{ }^{4} \mathrm{He}+{ }^{11} \mathrm{~B}={ }^{14} \mathrm{~N}+\mathrm{n},
$$

is less than $0.1 \%$ [20] of the number of $\mathrm{HB} 11$ reactions for the given spectrum of alphas as described [5, 6] after equation (1).

The neutron elimination device includes tin and is arranged such that the neutrons are brought to nuclear reactions with the tin. We suggest adding the tin that has proven to be particularly advantageous because of its high effective cross section [21], and the neutron reactions with tin transform the tin nuclei into stable nuclei with a higher atomic weight. The tin includes isotopes 114 to 119 and, with less than $0.01 \%$, isotopes 112 and 122 . The tin in purely metallic or compound form (e.g., alloy) absorbs the neutrons produced by the primary reaction or by secondary reactions in the reactor. The range of fast neutrons, which normally travel long distances through materials, is reduced if they have elastic collisions with protons or deuterons. This thermalization of fast neutrons is achieved with water or heavy water or with solid or liquid paraffin of sufficient thickness.

We can estimate the tin wall thickness needed for such a reactor in the following way: for a wall of length $x$, the relative number of neutrons going through the wall is calculated by

$$
\frac{N_{n}}{N_{n 0}}=e^{-(x / l)}=e^{-n_{0} \sigma x},
$$

where $N_{n 0}$ is the initial number of neutrons hitting the tin wall and $l$ is the mean free path for neutron absorption $=1$ / $\left(n_{0} \sigma\right)$, in which $n_{0}$ is the density number $\left(\mathrm{cm}^{-3}\right)$ of the tin medium absorbing the neutrons and $\sigma$ is the absorption cross section. The density of natural tin (Sn) in a solid state is 
$\rho=7.26 \mathrm{~g} / \mathrm{cm}^{3}$; therefore, we can calculate the number density to be $n_{0}=0.037 \times 10^{24} \mathrm{~cm}^{-3}$. As a typical example, we take a cross section $\sigma=6 b=6 \times 10^{-24} \mathrm{~cm}^{2}$ yielding

$$
-\ln \left(\frac{N_{n}}{N_{n 0}}\right)=0.22 x .
$$

Therefore, if we require $N_{n} / N_{n 0}=10^{-2}$ (i.e., $1 \%$ of the neutrons not absorbed), we get the tin wall thickness to be $x \approx 21 \mathrm{~cm}$. This estimation can be done for every relevant neutron energy.

A further advantage of the tin is the fact that no radiating residues remain.

\section{Data Availability}

The data can be made available upon request to the authors.

\section{Conflicts of Interest}

The authors declare no conflicts of interest.

\section{References}

[1] U. v. d. Leyen, Inauguration Speech European Council President, Portugal, 2019.

[2] J. Tennenbaum, 2020 Father of the Hydrogen-Boron Reactor, Asia Times, Hong Kong, China, 2020.

[3] J. P. Cockroft and E. T. S. Walton, "Experiments with high velocity positive ions. II-The disintegration of elements by high velocity protons," Proceedings of Royal Society of London A, vol. 137, p. 229, 1932.

[4] M. R. L. Oliphant and L. Rutherford, "Experiments on the transmutation of elements by protons," Proceedings of Royal Society London A, vol. 141, p. 259, 1932.

[5] V. E. Dimitriev, " $\alpha$ particle spectrum in reaction $\mathrm{p}+\mathrm{B} 11$ $\longrightarrow 3 \alpha$," Physics of Atomic Nuclei, vol. 72, p. 1165, 2009.

[6] D. H. H. Hoffmann, Private Communication, 2019.

[7] D. Strickland and G. Mourou, "Compression of amplified chirped optical pulses,” (1985) Optics Communication, vol. 56, 2019.

[8] V. S. Belyaev, A. P. Matafonov, V. I. Vinogradov, V. P. Krainov, and V. S. Lisitsa, "Observation of neutronless fusion reactions in picosecond laser plasmas," Physical Review E, vol. 72, p. 026406, 2006.

[9] C. Labaune, C. Baccou, S. Depierreux et al., "Fusion reactions initiated by laser-accelerated particle beams in a laser-produced plasma," Nature Communications, vol. 4, p. 2506, 2013.

[10] A. Picchiotto, D. Margarone, A. Velyhan et al., "Boron-proton nuclear fusion enhancement induced in boron-doped silicon targets by low-contrast pulsed laser," Physical Reviews X, vol. 4, p. 031030, 2014.

[11] L. Giuffrieda, F. Belloni, D. Margarone et al., "High-current stream of energetic $\alpha$ particles from laser-driven proton-boron fusion," Physical Review E, vol. 101, p. 013204, 2020.

[12] H. Hora, G. Korn, L. Giuffrida et al., "Fusion Energy using avalanche increased boron reactions for block-ignition by ultrahigh power picosecond laser pulses," Laser and Particle Beams, vol. 33, no. 4, p. 607, 2015.
[13] S. Eliezer, H. Hora, G. Korn, N. Nissim, and J. M. Martinez Val, "Avalanche proton-boron fusion based on elastic nuclear collisions,” Physics of Plasmas, vol. 23, p. 050708, 2016.

[14] S. V. Putvinski, D. D. Ryutov, and P. N. Yushmanov, "Fusion reactivity of the pB11 plasma revisited," Nuclear Fusion, vol. 59, p. 076018, 2019.

[15] W. M. Nevins and R. Swain, "The thermonuclear fusion rate coefficient for p-11B reactions," Nuclear Fusion, vol. 40, no. 4, p. $865,2000$.

[16] S. Eliezer and J. M. Martinez-Val, "A novel fusion reactor with chain reactions for proton-boron11," Laser and Particle Beams, vol. 38, no. 1, pp. 39-44, 2020.

[17] H. Hora, S. Eliezer, G. J. Kirchhoff et al., "Road map to clean energy using laser beam ignition of boron-hydrogen fusion," Laser and Particle Beams, vol. 35, no. 4, pp. 730-740, 2017.

[18] H. Hora, G. H. Miley, S. Eliezer, and N. Nissim, "Pressure of picosecond CPA laser pulses substitute ultrahigh thermal pressures to ignite fusion," High Energy Density Physics, vol. 35 , p. 10073, 2020.

[19] H. Hora, S. Eliezer, and N. A. Nissim, "Elimination of neutrons from nuclear reactions in reactor in particular clean laser boron-11 fusion without secondary contamination," United States Patent Application 20200321135, 2018.

[20] R. L. Walker, "The $(\alpha, n)$ cross section of boron," Physical Review, vol. 76, no. 2, p. 244, 1949.

[21] R. W. Harper, T. W. Godfrey, and J. L. Weil, "Interaction of neutrons with even-Atin isotopes. I. Total cross sections for En=0.3-5.0MeV," Physical Review C, vol. 26, no. 4, p. 1432, 1982. 